\title{
Formation of Organic-Inorganic Nanomatrix
}

2

3

4

5

6

7

8

9

10

11

12

\section{Structure with Nanosilica Networks and Its Effect on}

\author{
Properties of Rubber
}

Kenichiro Kosugi, Hitoshi Arai, Yuanbing Zhou, Seiichi Kawahara*

Department of Materials Science and Technology, Faculty of Engineering, Nagaoka University of Technology, 1603-1 Kamitomioka, Nagaoka, Niigata 940-2188, Japan

\section{*Corresponding Author}

E-mail: kawahara@mst.nagaokaut.ac.jp (S. Kawahara)

\section{ABSTRACT}

Graft-copolymerization of vinyl monomer containing silica precursor, $p$-styryltrimethoxysilane, onto natural rubber microparticles was performed in latex stage to form three-dimensional networks of nanosilica. After the latex was cast into a film, morphology observation by transmission electron microscopy showed that the natural rubber microparticles were dispersed in organic-inorganic nanomatrix consisting of vinyl polymer and silica nanoparticles. In particular, generation of large silica particles was prevented by slow rate of hydrolysis and condensation of silicon alkoxides for $p$-styryltrimethoxysilane and the nanosilica networks were formed. The nanomatrix structure with the nanosilica networks increased tensile strength of the 
1 rubber, and destruction of the structure decreased the strength. Master curves of dynamic moduli

2 of the rubber showed that the value of storage modulus at rubbery plateau region was high and it

3 was almost flat at low-frequency region because of the nanosilica networks.

\section{Keywords}

Nanocomposites; Filler network; Natural rubber

\section{Introduction}

Network-forming dispersion of inorganic nanofiller in polymer, which is distinguished from ordinary uniform dispersion, is required to enhance properties of polymeric nanocomposites,

11 because three-dimensional networks of the nanofiller result in the outstanding properties of the

12 nanocomposites. ${ }^{1-7}$ For instance, a modulus of the nanocomposites increases significantly when

13 the percolated networks of the nanofiller are formed. ${ }^{8-11}$ The nanofiller with higher surface area

14 is more effective in increasing the modulus of the materials because of its tendency to form

15 larger agglomerates that act as the filler networks. ${ }^{12-15}$ However, stress concentration during

16 deformation of the materials occurs in the vicinity of the large agglomerates of the filler,

17 initiating crack to reduce strength of the materials. ${ }^{16-18}$ Although preventing formation of the

18 large agglomerates of the nanofiller has been performed by reducing filler-filler interactions and

19 improving polymer-filler interactions, the resulting uniform dispersion of the nanofiller in

20 polymer is found to reduce the modulus. ${ }^{11,19-22}$ Therefore, in order to solve trade-off between the

21 modulus and strength of the polymeric nanocomposites, it is expected to establish a novel 22 concept for the network-forming dispersion of the nanofiller. 
The organic-inorganic nanomatrix structure is the novel concept to prepare the polymeric nanocomposites with the outstanding mechanical properties, in which polymeric microparticles are dispersed in organic-inorganic hybrid matrix with nanometers in thickness (nanomatrix). ${ }^{23}$ Because the inorganic nanofiller is condensed in the nanomatrix to form the three-dimensional networks of the nanofiller, the organic-inorganic nanomatrix structure may be effective in enhancing both the modulus and strength. This structure may be formed by graft-copolymerization of vinyl monomer containing metal alkoxide (precursor) onto the polymeric microparticles in a latex stage, where hydrolysis and condensation of the metal alkoxide occur to generate the nanofiller. Casting the resulting latex into a film, we may obtain the polymeric microparticles dispersed in the organic-inorganic nanomatrix that consists of the vinyl polymer and the nanofiller as the filler networks. The vinyl polymer may improve the interfacial strength between the polymeric microparticles and nanofiller, which is also an important factor to improve the properties of the polymeric nanocomposites.

Design of a polymerization system is crucial to form the organic-inorganic nanomatrix structure with the three-dimensional networks of the nanofiller. In the previous works, ${ }^{23,24}$ we performed graft-copolymerization of vinyltriethoxysilane (VTES) onto natural rubber (NR) microparticles in the latex stage. After casting the latex into the film, it was found that the NR microparticles were dispersed in the nanomatrix consisting of poly(VTES) and silica nanoparticles that were generated by the hydrolysis and condensation of silicon alkoxide. However, undesirable silica particles with diameter of larger than $200 \mathrm{~nm}$ were also generated, which induced coagulation of the latex during the polymerization and reduced the mechanical properties of the rubber. Furthermore, since almost monomer was converted to the large silica particles, the thickness of the nanomatrix was so thin that the effect of the nanomatrix on the 
1 modulus of the rubber was small. It is, thus, required to prevent generation of the large silica

2 particles by controlling the hydrolysis and condensation of the silicon alkoxide. Because bulky

3 group such as phenyl group attached to the silicon atom suppresses the hydrolysis via steric

4 hindrance, ${ }^{25,26}$ using $p$-styryltrimethoxysilane (STMS) as the monomer may yield the

5 three-dimensional networks of the nanosilica without generation of the large silica particles.

6 In the present study, we performed the graft-copolymerization of STMS onto the NR

7 microparticles in the latex stage using tert-butyl hydroperoxide (TBHPO) and

8 tetraethylenepentamine (TEPA) as an initiator. The resulting latex of the grafted rubber

9 (NR-graft-PSTMS) was cast into the film to form the organic-inorganic nanomatrix structure

10 with the three-dimensional networks of the nanosilica. Suitable monomer and initiator

11 concentrations were determined in relation to conversion of monomer together with morphology

12 of the as-cast film. The effect of the three-dimensional networks of the nanosilica on the

13 mechanical and viscoelastic properties of the rubber was studied.

\section{Experimental section}

16

\subsection{Materials}

Commercial high ammonia NR (HANR) latex, which contained about 60 w/w\% dry rubber, was used as a source of the NR latex. Deproteinization of the HANR latex with urea in the presence of a surfactant was carried out according to the literature ${ }^{27}$ to prevent a side reaction due to proteins present in the HANR latex. The HANR latex was diluted with aqueous solution of sodium dodecyl sulfate (SDS, Kishida Chemical, 98 w/w\%) so that the dry rubber content and SDS concentration in the latex were $30 \mathrm{w} / \mathrm{w} \%$ and $1 \mathrm{w} / \mathrm{w} \%$, respectively, and then $0.1 \mathrm{w} / \mathrm{w} \%$ urea (Nacalai Tesque, $99.0 \mathrm{w} / \mathrm{w} \%$ ) was added to the latex. The latex was incubated at ambient 
temperature for $1 \mathrm{~h}$ by gently stirring and then centrifuged at 10,000 $\mathrm{g}$ at $288 \mathrm{~K}$ for $30 \mathrm{~min}$. The cream fraction was re-dispersed in $0.5 \mathrm{w} / \mathrm{w} \%$ SDS solution, and it was washed twice by centrifugation to prepare deproteinized NR latex. The resulting deproteinized NR latex was adjusted to about $20 \mathrm{w} / \mathrm{w} \%$ dry rubber content and $0.5 \mathrm{w} / \mathrm{w} \%$ SDS concentration.

The graft-copolymerization of STMS onto the deproteinized NR particles was carried out in the latex stage with TBHPO and TEPA as the initiator in the way similar to that reported in the previous work. ${ }^{23,24}$ We used TBHPO (Kishida Chemical, 68 w/w\%), TEPA (Kishida Chemical, 95 w/w\%), and STMS (Shin-Etsu Silicone KBM-1403) as received. The deproteinized NR latex was charged with $\mathrm{N}_{2}$ gas at $303 \mathrm{~K}$ for $1 \mathrm{~h}$, and then TBHPO and TEPA were added to the latex followed by addition of STMS. The graft-copolymerization was performed by stirring the latex at $200 \mathrm{rpm}$ at $303 \mathrm{~K}$ for $2 \mathrm{~h}$. The resulting latex was poured into a petri dish and dried in an oven at $323 \mathrm{~K}$. The film with about $1 \mathrm{~mm}$ in thickness thus formed was further dried under vacuum at $323 \mathrm{~K}$ for several days. A film from the deproteinized NR latex was also prepared in the similar way.

In order to remove unreacted monomer that remained in the as-cast film of NR-graft-PSTMS, the film was extracted with acetone in a Soxhlet apparatus under $\mathrm{N}_{2}$ atmosphere in the dark for $24 \mathrm{~h}$ and then dried under vacuum for a week. Content and conversion of monomer were determined by gravimetric method after extraction of the unreacted monomer. Because some non-rubber components contained in NR such as fatty acids are known to be removed by acetone extraction, ${ }^{28}$ the as-cast film from the deproteinized NR latex was also extracted with acetone.

\subsection{Measurements}

The morphology was observed by transmission electron microscopy (TEM) with a JEOL 
1 JEM-2100 at accelerating voltage of $200 \mathrm{kV}$ without staining the samples. Ultrathin sections of

2 the film were prepared with a Reichert-Nissei ULTRACUT N/FC S at $183 \mathrm{~K}$.

3 The tensile properties of the film were measured with an Instron 3365 by uniaxially stretching

4 a Dumbell-shaped (JIS K6251 Type 7) specimen at crosshead speed of $200 \mathrm{~mm} \mathrm{~min}^{-1}$ at ambient

5 temperature.

6 Dynamic mechanical properties were measured with an Anton Paar Physica MCR 301 using 7 a parallel-plate geometry of $12 \mathrm{~mm}$ diameter. The dynamic shear moduli were obtained at

8 temperatures between 203 and $423 \mathrm{~K}$ and frequencies between 0.1 to $10 \mathrm{~s}^{-1}$. The oscillatory

9 strain amplitudes were within the range of linear viscoelasticity in all measurements. Master

10 curves of the dynamic moduli were obtained by time-temperature superpositioning of the moduli

11 with reference temperature of $208 \mathrm{~K}$ in the following manner. ${ }^{6}$ First, loss tangent ( $\tan \delta$ ) curves

12 were shifted horizontally to determine horizontal shift factor $\left(a_{T}\right)$. Second, storage modulus $\left(G^{\prime}\right)$

13 and loss modulus $\left(G^{\prime \prime}\right)$ curves were shifted horizontally using determined $a_{T}$ and then they were

14 shifted vertically to determine vertical shift factor $\left(b_{T}\right)$, in which the value of $b_{T}$ for $G^{\prime}$ and $G^{\prime \prime}$

15 were the same.

16

17 Table 1. Graft-Copolymerization of p-Styryltrimethoxysilane onto Natural Rubber 18 Microparticles in Latex Stage ${ }^{a}$

\begin{tabular}{ccccc}
\hline Sample code & $\begin{array}{c}\text { Monomer } \\
\text { [mol/kg-rubber] }\end{array}$ & $\begin{array}{c}\text { Initiator }^{b} \\
\text { [mol/kg-rubber] }\end{array}$ & $\begin{array}{c}\text { Conversion }^{c} \\
\text { [w/w\%] }\end{array}$ & $\begin{array}{c}\text { Monomer Content } \\
\text { [w/w\%] }\end{array}$ \\
\hline NR-graft-PSTMS_1.0_3.3 & 1.0 & $3.3 \times 10^{-2}$ & 27.7 & 5.1 \\
NR-graft-PSTMS_1.0_6.6 & 1.0 & $6.6 \times 10^{-2}$ & 52.3 & 9.6 \\
NR-graft-PSTMS_1.0_16.5 & 1.0 & $16.5 \times 10^{-2}$ & 30.3 & 5.6 \\
NR-graft-PSTMS_0.5_3.3 & 0.50 & $3.3 \times 10^{-2}$ & 51.5 & 4.7 \\
NR-graft-PSTMS_0.5_6.6 & 0.50 & $6.6 \times 10^{-2}$ & 25.5 & 2.3 \\
\hline
\end{tabular}


${ }^{a}$ At $303 \mathrm{~K}$ for $2 \mathrm{~h}$ using deproteinized natural rubber latex with $20 \mathrm{w} / \mathrm{w} \%$ dry rubber content. ${ }^{b}$ tert-butyl hydroperoxide and tetraethylenepentamine. ${ }^{c}$ Determined by gravimetric method.

\section{Results and discussion}

The condition and conversion for the graft-copolymerization of STMS onto the deproteinized NR microparticles in the latex stage are shown in Table 1 in conjunction with STMS content of NR-graft-PSTMS. The relatively high conversion was achieved with the initiator concentration of $6.6 \times 10^{-2} \mathrm{~mol} / \mathrm{kg}$-rubber for the monomer concentration of $1.0 \mathrm{~mol} / \mathrm{kg}$-rubber (NR-graft-PSTMS_1.0_6.6) and with the initiator concentration of $3.3 \times 10^{-2} \mathrm{~mol} / \mathrm{kg}$-rubber for the monomer concentration of $0.5 \mathrm{~mol} / \mathrm{kg}$-rubber (NR-graft-PSTMS_0.5_3.3). Note that the conversion did not increase with increasing the initiator concentration and also the highest conversion was only about $50 \mathrm{w} / \mathrm{w} \%$. These results were distinguished from those for the graft-copolymerization with VTES under the similar condition; that is, the conversion for VTES increased with increasing the initiator concentration and was more than $98 \mathrm{w} / \mathrm{w} \%$ at the initiator concentration of $6.6 \times 10^{-2} \mathrm{~mol} / \mathrm{kg}$-rubber. ${ }^{24}$ This difference between STMS and VTES may be explained on the basis of the difference in the reactivity of the silicon alkoxide. For VTES, the rate of the hydrolysis and condensation of the silicon alkoxide was fast enough to convert almost all of the monomer to the silica particles, especially at the high initiator concentration because TEPA used as the initiator made the latex alkaline to promote the hydrolysis and condensation. On the other hand, the rate of the hydrolysis and condensation for STMS was slow because of the bulky phenyl group on the silicon atom even under the alkaline condition. The decrease in the conversion at high initiator concentration may be due to chain transfer to the excess initiator, which was reported in the graft-copolymerization of styrene onto the NR microparticles. ${ }^{29}$ 
1 Consequently, it was indicated that the hydrolysis and condensation of the silicon alkoxide were

2 suppressed for STMS relative to VTES; thus, generaation of the large silica particles may be

3 prevented. Hereafter, we focus our study on the sample with the relatively high conversion,

4 namely, NR-graft-PSTMS_1.0_6.6 and NR-graft-PSTMS_0.5_3.3, in which the monomer

5 content was $9.6 \mathrm{w} / \mathrm{w} \%$ and $4.7 \mathrm{w} / \mathrm{w} \%$, respectively.

6

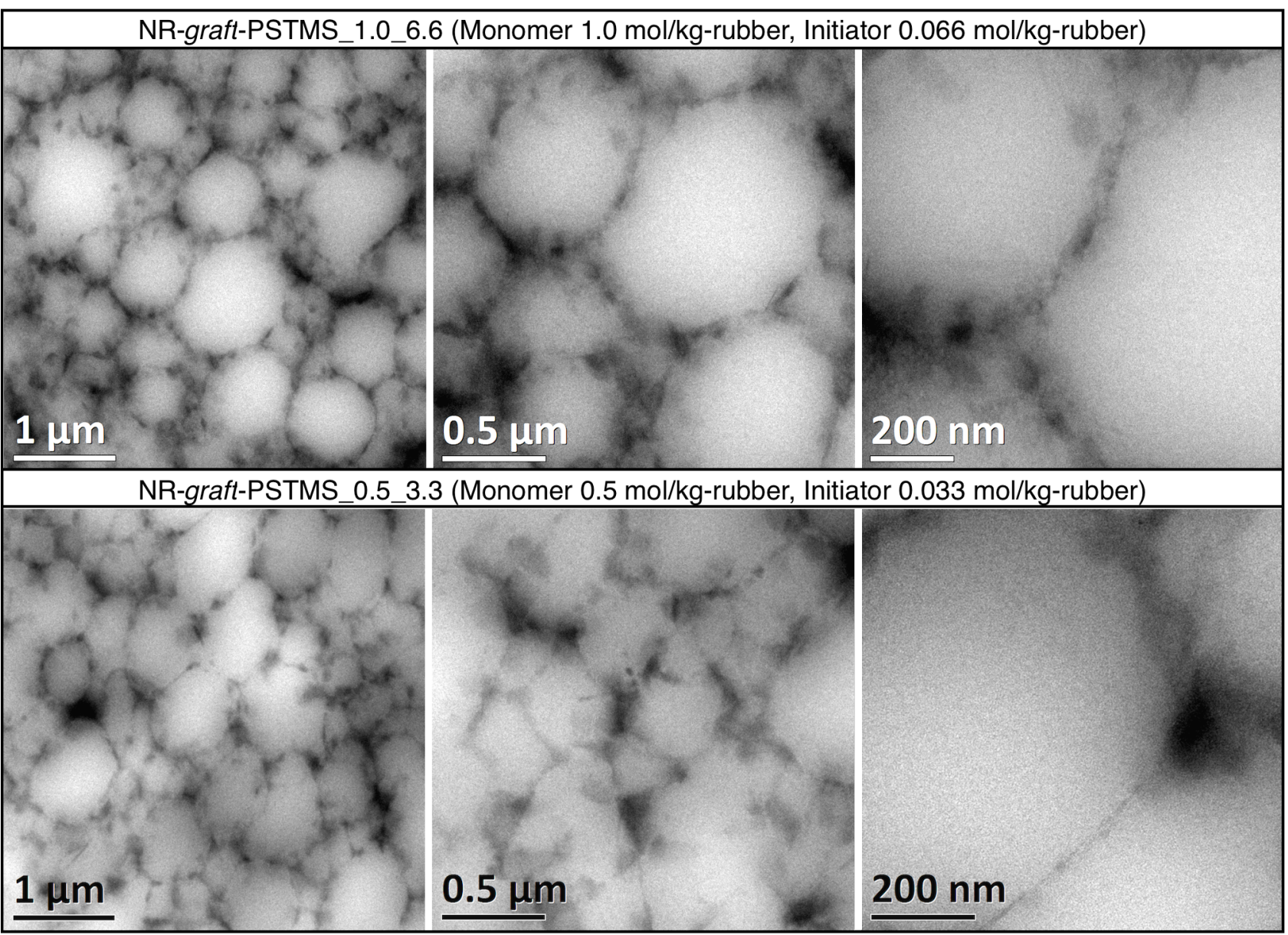

8 Figure 1. TEM images for as-cast film of natural rubber grafted with

9 poly(p-styryltrimethoxysilane) (NR-graft-PSTMS): top， NR-graft-PSTMS_1.0_6.6; bottom,

10 NR-graft-PSTMS_0.5_3.3. Because of non-staining, bright domain represents natural rubber

11 microparticles and dark domain silica nanoparticles. 
2 The nanosilica networks formed in the as-cast film of NR-graft-PSTMS_1.0_6.6 and

3 NR-graft-PSTMS_0.5_3.3 were observed by TEM, as shown in Figure 1, in which bright

4 domain represents the NR microparticles and dark domain the silica nanoparticles because the

5 sample was not stained. In the TEM images, the NR particles with about $1 \mu \mathrm{m}$ in average

6 diameter were dispersed in the nanomatrix consisting of PSTMS and the silica nanoparticles that

7 formed the three-dimensional networks. The thickness of the nanomatrix increased with

8 increasing the monomer content of NR-graft-PSTMS; that is, the thickness for

9 NR-graft-PSTMS_1.0_6.6 was thicker than that for NR-graft-PSTMS_0.5_3.3. It should be

10 emphasized that the large silica particles with diameter of more than $200 \mathrm{~nm}$ were not generated

11 in this study, although they were generated as VTES was used as the monomer. ${ }^{23,24}$ The

12 graft-copolymerization of VTES did not form the silica networks, since the large silica particles

13 were generated in NR-graft-PVTES. In contrast, STMS formed the nanosilica networks, since

14 the silica nanoparticles were generated. This difference between STMS and VTES may be

15 explained due to the difference in the rate of the hydrolysis and condensations of the silicon

16 alkoxide; thus, the slower rate for STMS as compared with VTES suppressed the generation of

17 the large silica particles.

18 Because the unreacted monomer and homopolymer may remain in the as-cast film of

19 NR-graft-PSTMS, the film was extracted with acetone (good solvent for STMS) and the TEM

20 observation was performed as shown in Figure 2. For NR-graft-PSTMS_1.0_6.6, the nanomatrix

21 disappeared; so that, fusion of the NR microparticles took place. In contrast,

22 NR-graft-PSTMS_0.5_3.3 possessed the nanomatrix as the nanosilica networks even after the

23 extraction. These results suggested that the nanomatrix of NR-graft-PSTMS_1.0_6.6 consisted 
1 of unreacted monomer or homopolymer whereas the nanomatrix of NR-graft-PSTMS_0.5_3.3

2 consisted of mainly grafted PSTMS. Consequently, although higher monomer concentration

3 yielded higher monomer content, the monomer concentration of $0.5 \mathrm{~mol} / \mathrm{kg}$-rubber and the 4 initiator concentration of $3.3 \times 10^{-2} \mathrm{~mol} / \mathrm{kg}$-rubber (NR-graft-PSTMS_0.5_3.3) were suitable to 5 form the organic-inorganic nanomatrix structure with the nanosilica networks.

6

7

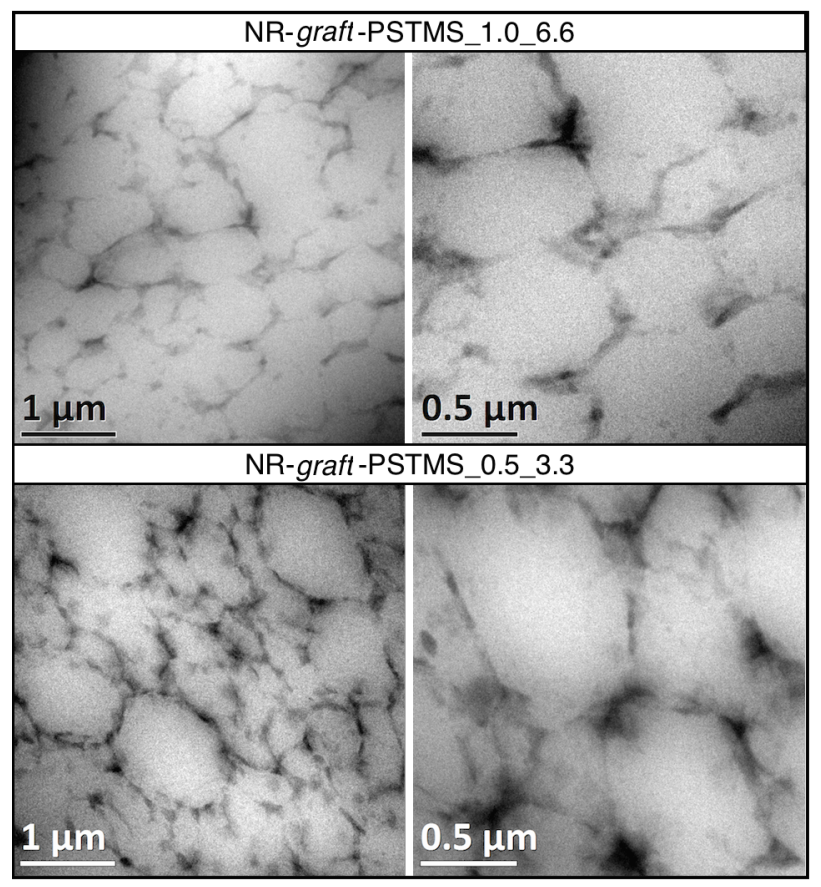

8 Figure 2. TEM images for acetone-extracted film of natural rubber grafted with 9 poly(p-styryltrimethoxysilane) (NR-graft-PSTMS): top, NR-graft-PSTMS_1.0_6.6; bottom, 10 NR-graft-PSTMS_0.5_3.3. 
5

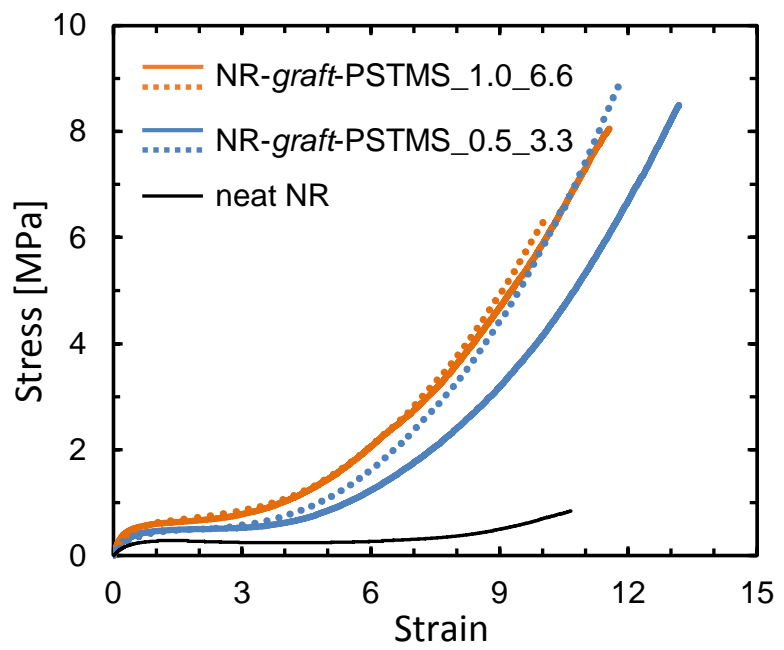

Figure 3. Nominal stress-strain curves for as-cast film (solid line) and acetone extracted film (dotted line) of natural rubber grafted with poly(p-styryltrimethoxysilane) (NR-graft-PSTMS) and for neat NR (thin line).

The stress-strain curves for the as-cast film of NR-graft-PSTMS and for neat NR (deproteinized NR after acetone extraction) are shown in Figure 3. The formation of the organicinorganic nanomatrix structure improved both the tensile strength and stress at initial strain for the rubber. The stress at initial strain, which corresponds to the modulus, increased with increasing the monomer content of NR-graft-PSTMS; that is, NR-graft-PSTMS_1.0_6.6 with 9.6 w/w\% monomer content showed higher stress at initial strain than that for NR-graft-PSTMS_0.5_3.3 with 4.7 w/w\% monomer content. On the other hand, the tensile strength for NR-graft-PSTMS_0.5_3.3 (8.5 MPa) was slightly higher than that for NR-graft-PSTMS_1.0_6.6 (8.1 MPa) although the monomer content of the former was half as low as the content of the latter. Therefore, the enhancement of the tensile strength by formation of the organic-inorganic nanomatrix structure may be due to not only the volume effect of the silica nanoparticles but also the effect of the nanomatrix as the nanosilica networks. In order to 
1 study the effect of the nanomatrix on the strength, we performed the tensile test for the

acetone-extracted film of NR-graft-PSTMS. As shown in Figure 3, the value of the tensile strength for NR-graft-PSTMS_1.0_6.6 decreased from 8.1 to 6.3 MPa by the extraction, whereas that for NR-graft-PSTMS_0.5_3.3 did not change. Based on the fact that the destruction of the nanomatrix structure by the acetone extraction was observed for NR-graft-PSTMS_1.0_6.6 but not for NR-graft-PSTMS_0.5_3.3 as shown in Figure 2, the decrease in the tensile strength for NR-graft-PSTMS_1.0_6.6 by the extraction was attributed to the destruction of the nanomatrix structure. Accordingly, the tensile strength for NR-graft-PSTMS with the organic-inorganic nanomatrix structure was found to be dependent upon the morphology and it was about 10 times higher than that for the neat NR when the nanosilica networks were formed.

The viscoelastic properties over the wide-frequency region for NR-graft-PSTMS with the organic-inorganic nanomatrix structure were studied by constructing master curves of dynamic moduli. Figure 4 shows the master curves of $G^{\prime}$ and $G^{\prime \prime}$ for the acetone-extracted film of NR-graft-PSTMS_0.5_3.3 and neat NR. The master curves for neat NR showed typical viscoelastic properties of uncrosslinked rubber, in which the value of $G^{\prime}$ at rubbery plateau region was about $10^{5} \mathrm{~Pa}$. The flow behavior was observed at low-frequency region, although transition from the rubbery plateau to the flow zone was not sharp and slopes of $G^{\prime}$ and $G^{\prime \prime}$ curves at the flow zone were not 2 and 1 , respectively, because of wide molecular weight distribution and branching structure of $\mathrm{NR}^{28}$ After forming the organic-inorganic nanomatrix structure with the nanosilica networks, the viscoelastic properties of the rubber changed slightly at high-frequency region and dramatically at low-frequency region. The peak of $G^{\prime \prime}$ curve at high-frequency region, which corresponds to segmental relaxation of polymer, for NR-graft-PSTMS_0.5_3.3 was slightly broader than that for neat NR. This broadening of $G^{\prime \prime}$ 
1 peak was reported for conventional filled rubber and ascribed to slowed-down segmental

2 dynamics of polymer in close proximity to the filler surface. ${ }^{30,31}$ On the other hand, at

3 low-frequency region the master curve of $G^{\prime}$ for NR-graft-PSTMS_0.5_3.3 showed higher and

4 longer rubbery plateau region as compared with that for neat NR. The value of $G^{\prime}$ at the plateau

5 region (for example, at $\omega a_{T}=10^{-7}$ ) for NR-graft-PSTMS_0.5_3.3 was 4 times higher than that

6 for neat NR. The 4 times increase for NR-graft-PSTMS_0.5_3.3 with 4.7 w/w\% monomer

7 content was much higher than that approximated by Guth-Gold equation, ${ }^{32}$ which expresses the

8 increase in the modulus by a volume (hydrodynamic) effect of the filler:

$$
G^{\prime}=G_{0}^{\prime}\left(1+2.5 \phi+14.1 \phi^{2}\right)
$$

10 where $\phi$ is volume fraction of the filler and $G^{\prime}$ and $G_{0}^{\prime}$ are the storage moduli of the filled and 11 neat rubber, respectively. In addition, when we used VTES as the monomer in the previous 12 works, the value of $G^{\prime}$ for the grafted rubber was only 2 times higher than that for neat NR 13 although the monomer content was more than $10 \mathrm{w} / \mathrm{w} \%{ }^{24}$ The high value of $G^{\prime}$ for 14 NR-graft-PSTMS_0.5_3.3 may be attributed to the formation of the organic-inorganic nanomatrix structure with the nanosilica networks. The existence of the nanosilica networks for NR-graft-PSTMS_0.5_3.3 was further supported by suppression of the flow behavior at low-frequency region, where the curve of $G^{\prime}$ were nearly flat and that of $G^{\prime \prime}$ increased with decreasing the frequency. The value of $G^{\prime}$ was higher than that of $G^{\prime \prime}$, indicating that the elastic networks of the nanosilica were formed in NR-graft-PSTMS_0.5_3.3. 

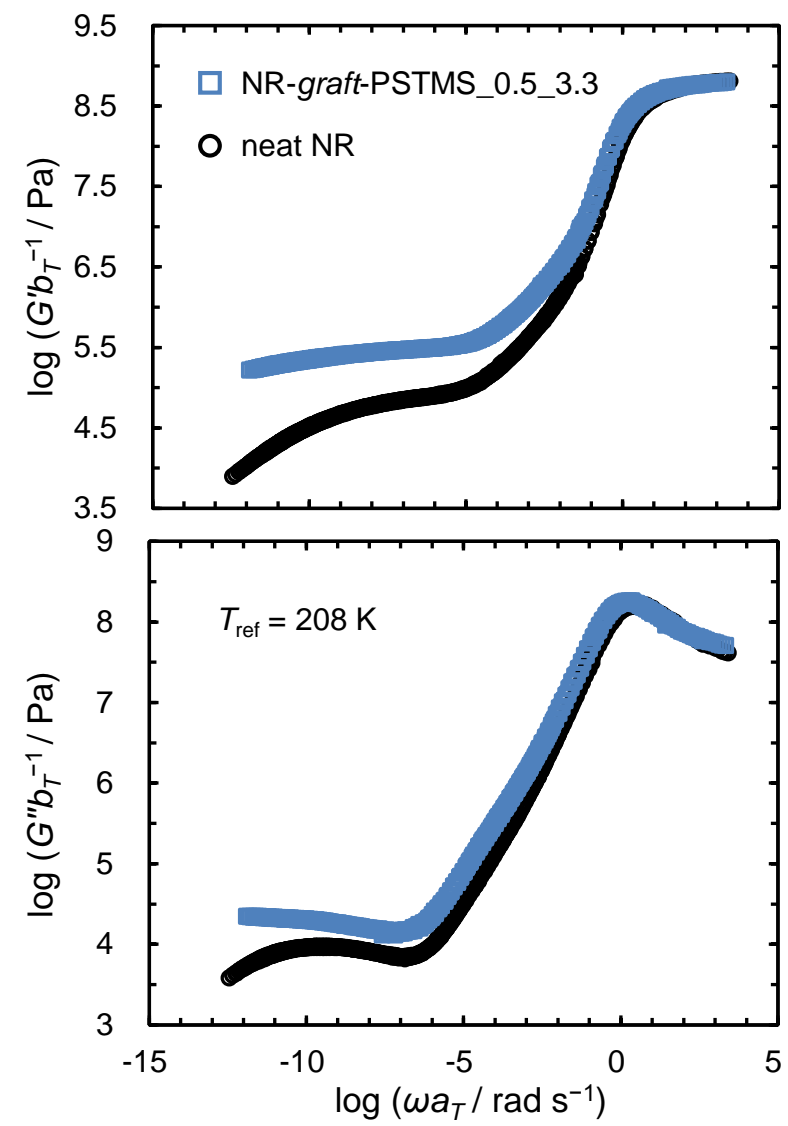

Figure 4. Master curves of storage modulus (top) and loss modulus (bottom) for

3 acetone-extracted film of natural rubber grafted with poly(p-styryltrimethoxysilane)

4 (NR-graft-PSTMS) and for neat NR. Monomer content of NR-graft-PSTMS was 4.7 w/w\%.

\section{Conclusions}

The graft-copolymerization of STMS was performed onto the deproteinized NR

8 microparticles with TBHPO and TEPA as the initiator in the latex stage. At the initiator

9 concentration of $3.3 \times 10^{-2} \mathrm{~mol} / \mathrm{kg}$-rubber and the monomer concentration of $0.5 \mathrm{~mol} / \mathrm{kg}$-rubber

10 (NR-graft-PSTMS_0.5_3.3), the conversion and content of the monomer were 51.5 and 4.7

11 w/w\%, respectively. The TEM images for the as-cast film of NR-graft-PSTMS_0.5_3.3 showed

12 that the NR particles with about $1 \mu \mathrm{m}$ in average diameter were dispersed in the nanomatrix 


\section{Acknowledgment}

\section{References} 265-271.

consisting of PSTMS and the silica nanoparticles, even after the acetone extraction. The tensile strength for the as-cast film of NR-graft-PSTMS_0.5_3.3 was $8.5 \mathrm{MPa}$, which was 10 times higher than that for neat NR, and it did not change after the extraction. The master curves of the dynamic moduli for acetone-extracted NR-graft-PSTMS_0.5_3.3 showed that the value of $G^{\prime}$ at rubbery plateau region was 4 times higher than that for neat NR and it was almost flat at low-frequency region, indicating that the elastic networks of the nanosilica were formed in NR-graft-PSTMS_0.5_3.3.

This work was supported in part by a Grant-in-Aid (21655080) for Challenging Exploratory Research and Grant-in-Aid (223501000) for Scientific Research (B) from Japan Society for the Promotion of Science and JST-JICA SATREPS.

(1) J. G. Meier, M. Klüppel, Carbon Black Networking in Elastomers Monitored by Dynamic Mechanical and Dielectric Spectroscopy, Macromol. Mater. Eng. 2008, 293 12-38.

(2) M. Sumita, K. Sakata, S. Asai, K. Miyasaka, H. Nakagawa, Dispersion of Fillers and the Electrical Conductivity of Polymer Blends Filled with Carbon Black, Polym. Bull. 1991, 25,

(3) Z. Zhu, T. Thompson, S. -Q. Wang, E. D. Von Meerwall, A. Halasa, Investigating Linear and Nonlinear Viscoelastic Behavior Using Model Silica-Particle-Filled Polybutadiene, Macromolecules 2005, 38, 8816-8824. 
1 (4) N. Jouault, P. Vallat, F. Dalmas, S. Said, J. Jestin, F. Boué, Well-Dispersed Fractal Aggregates as Filler in Polymer-Silica Nanocomposites: Long-Range Effects in Rheology, Macromolecules 2009, 42, 2031-2040.

4

(5) G. Filippone, G. Romeo, D. Acierno, Viscoelasticity and Structure of Polystyrene/Fumed 2714-2720.

(6) Y. Isono, T. Aoyama, Filler Effects on Temperature Shift Factors in Viscoelastic Properties of Carbon Black Filled Rubbers, Nihon Reoroji Gakkaishi 2013, 41, 137-144.

(7) L. Chen, W. Zhou, J. Lu, J. Li, W. Zhang, N. Huang, L. Wu, L. Li, Unveiling Reinforcement and Toughening Mechanism of Filler Network in Natural Rubber with Synchrotron Radiation X-ray Nano-Computed Tomography, Macromolecules 2015, 48, 7923-7928.

(8) A. R. Payne, The Dynamic Properties of Carbon Black-Loaded Natural Rubber Vulcanizates. Part I, J. Appl. Polym. Sci. 1962, 6, 57-63.

(9) K. Yurekli, R. Krishnamoorti, M. F. Tse, K. O. Mcelrath, A. H. Tsou, H. -C. Wang, Structure and Dynamics of Carbon Black-Filled Elastomers, J. Polym. Sci. Partt B: Polym. Phys. 2001, 39, 256-275.

(10)G. Raos, M. Moreno, S. Elli, Computational Experiments on Filled Rubber Viscoelasticity: What Is the Role of Particle-Particle Interactions?, Macromolecules 2006, 39, 6744-6751.

(11)C. G. Robertson, C. J. Lin, R. B. Bogoslovov, M. Rackaitis, P. Sadhukhan, J. D. Quinn, C. M. Roland, Flocculation, Reinforcement, and Glass Transition Effects in Silica-Filled Styrene-Butadiene Rubber, Rubber Chem. Technol. 2011, 84, 507-519.

(12)F. Yatsuyanagi, N. Suzuki, M. Ito, H. Kaidou, Effects of Secondary Structure of Fillers on the Mechanical Properties of Silica Filled Rubber Systems, Polymer 2001, 42, 9523-9529. 
1 (13)J. Oberdisse, Structure and Rheological Properties of Latex-Silica Nanocomposite Films:

2 Stress-Strain Isotherms, Macromolecules 2002, 35, 9441-9450.

3 (14)C. G. Robertson, C. J. Lin, M. Rackaitis, C. M. Roland, Influence of Particle Size and

4 Polymer-Filler Coupling on Viscoelastic Glass Transition of Particle-Reinforced Polymers, 5 Macromolecules 2008, 41, 2727-2731.

6 (15)N. Jouault, F. Dalmas, F. Boué, J. Jestin, Multiscale Characterization of Filler Dispersion 7 and Origins of Mechanical Reinforcement in Model Nanocomposites, Polymer 2012, 53, 7618775.

9 (16)A. N. Gent, B. Park, Failure Processes in Elastomers at or Near a Rigid Spherical Inclusion, 10 J. Mater. Sci. 1984, 19, 1947-1956.

11 (17)A. N. Gent, Y. -C. Hwang, Internal Failures in Model Elastomeric Composites, J. Mater. 12 Sci. 1990, 25, 4981-4986.

13 (18)K. Akutagawa, K. Yamaguchi, A. Yamamoto, H. Heguri, H. Jinnai, Y. Shinbori, 14 Mesoscopic Mechanical Analysis of Filled Elastomer with 3D-Finite Element Analysis and 15 Transmission Electron Microtomography, Rubber Chem. Technol. 2008, 81, 182-189.

16 (19)F. Yatsuyanagi, N. Suzuki, M. Ito, H. Kaidou, Effects of Surface Chemistry of Silica 17 Particles on the Mechanical Properties of Silica Filled Styrene-Butadiene Rubber Systems, 18 Polym. J. 2002, 34, 332-339.

19 (20)Y. Ikeda, Y. Kameda, Preparation of "Green" Composites by the Sol-Gel Process: In Situ 20 Silica Filled Natural Rubber, J. Sol-Gel Sci. Technol., 2004, 31, 137-142.

21 (21)C. Gauthier, E. Reynaud, R. Vassoille, L. Ladouce-Stelandre, Analysis of the Non-Linear 22 Viscoelastic Behaviour of Silica Filled Styrene Butadiene Rubber, Polymer 2004, 45, 2761$23 \quad 2771$. 
1 (22)X. Liu, S. Zhao, X. Zhang, X. Li, Y. Bai, Preparation, Structure, and Properties of

2 Solution-Polymerized Styrene-Butadiene Rubber with Functionalized End-Groups and Its

3 Silica-Filled Composites, Polymer 2014, 55, 1964-1976.

4 (23) S. Kawahara, N. H. Yusof, K. Noguchi, K. Kosugi, Y. Yamamoto, Organic-Inorganic

5 Nanomatrix Structure and Properties of Related Naturally Occurring Rubbery Macromolecules,

$6 \quad$ Polymer 2014, 55, 5024-5027.

7 (24)N. H. Yusof, K. Noguchi, L. Fukuhara, Y. Yamamoto, S. Kawahara, Preparation and

8 Properties of Natural Rubber with Filler Nanomatrix Structure, Colloid Polym. Sci. 2015, 293, $9 \quad 2249-2256$.

10 (25)R. C. Chambers, W. E. Jones Jr., Y. Haruvy, S. E. Webber, M. A. Fox, Influence of Steric 11 Effects on the Kinetics of Ethyltrimethoxysilane Hydrolysis in a Fast Sol-Gel System, Chem. 12 Mater. 1993, 5, 1481-1486.

13 (26)D. A. Loy, B. M. Baugher, C. R. Baugher, D. A. Schneider, K. Rahimian, Substituent 14 Effects on the Sol-Gel Chemistry of Organotrialkoxysilanes, Chem. Mater. 2000, 12, 3624153632.

16 (27)O. Chaikumpollert, Y. Yamamoto, K. Suchiva, S. Kawahara, Protein-Free Natural Rubber, 17 Colloid Polym. Sci. 2012, 290, 331-338.

18 (28) Y. Tanaka, Structural Characterization of Natural Polyisoprenes: Solve the Mystery of 19 Natural Rubber based on Structural Study, Rubber Chem. Technol. 2001, 74, 355-375.

20 (29)N. Pukkate, Y. Yamamoto, S. Kawahara, Mechanism of Graft Copolymerization of Styrene 21 onto Deproteinized Natural Rubber, Colloid Polym. Sci. 2008, 286, 411-416.

22 (30)J. H. Roh, M. Tyagi, T. E. Hogan, C. M. Roland, Space-Dependent Dynamics in 23 1,4-Polybutadiene Nanocomposite, Macromolecules 2013, 46, 6667-6669. 
1 (31)M. Klüppel, Evaluation of Viscoelastic Master Curves of Filled Elastomers and Applications

2 to Fracture Mechanics, J. Phys. Condens. Matter 2009, 21, 035104.

3 (32)E. Guth, Theory of Filler Reinforcement, J. Appl. Phys.1945, 16, 20-25. 
Graphical Abstract

Formation of Three-Dimensional Networks of Nanosilica
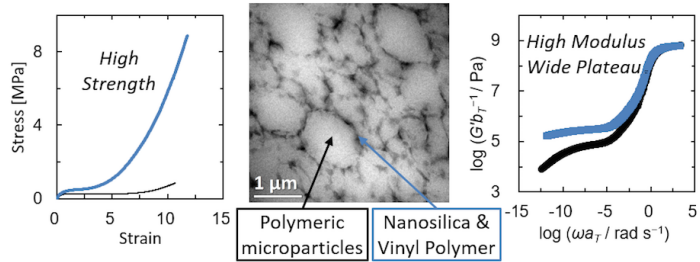